\title{
Programming for Stimulation-Induced Transient Nonmotor Psychiatric Symptoms after Bilateral Subthalamic Nucleus Deep Brain Stimulation for Parkinson's Disease
}

\author{
Xi Wu, ${ }^{1}$ Yiqing Qiu, ${ }^{1}$ Keith Simfukwe, ${ }^{2}$ Jiali Wang, ${ }^{1}$ Jianchun Chen, ${ }^{1}$ and Xiaowu Hu ${ }^{1}$ \\ ${ }^{1}$ Department of Neurosurgery, Second Military Medical University, Changhai Hospital, No. 168 Changhai Road, Yangpu District, \\ Shanghai, China \\ ${ }^{2}$ Department of Neurosurgery, Changhai Hospital, Second Military Medical University, International College of Exchange, \\ No. 800 Xiangyin Road, Shanghai 200433, China \\ Correspondence should be addressed to Xiaowu Hu; huxiaowu_smmu@sina.com
}

Received 24 April 2017; Revised 21 June 2017; Accepted 10 July 2017; Published 15 August 2017

Academic Editor: Hélio Teive

Copyright (C) $2017 \mathrm{Xi} \mathrm{Wu}$ et al. This is an open access article distributed under the Creative Commons Attribution License, which permits unrestricted use, distribution, and reproduction in any medium, provided the original work is properly cited.

\begin{abstract}
Background. Stimulation-induced transient nonmotor psychiatric symptoms (STPSs) are side effects following bilateral subthalamic nucleus deep brain stimulation (STN-DBS) in Parkinson's disease (PD) patients. We designed algorithms which (1) determine the electrode contacts that induce STPSs and (2) provide a programming protocol to eliminate STPS and maintain the optimal motor functions. Our objective is to test the effectiveness of these algorithms. Materials and Methods. 454 PD patients who underwent programming sessions after STN-DBS implantations were retrospectively analyzed. Only STPS patients were enrolled. In these patients, the contacts inducing STPS were found and the programming protocol algorithms used. Results. Eleven patients were diagnosed with STPS. Of these patients, two had four episodes of crying, and two had four episodes of mirthful laughter. In one patient, two episodes of abnormal sense of spatial orientation were observed. Hallucination episodes were observed twice in one patient, while five patients recorded eight episodes of hypomania. There were no statistical differences between the UPDRS-III under the final stimulation parameter (without STPS) and previous optimum UPDRS-III under the STPSs $(p=1.000)$. Conclusion. The flow diagram used for determining electrode contacts that induce STPS and the programming protocol employed in the treatment of these symptoms are effective.
\end{abstract}

\section{Introductions}

Subthalamic nucleus deep brain stimulation (STN-DBS) is an effective therapy which ameliorates motor manifestations suffered by patients with idiopathic Parkinson's disease (PD). The hallmark symptoms in PD patients include tremor, rigidity, and bradykinesia. STN-DBS has been documented to be well tolerated by PD patients with marked improvement of motor functions even after over ten-year follow-up [1-3]. The limbic system innervates the limbic part of STN and other anatomic surrounding structures that lay in proximity [4]. As a result, the likelihood of accidental tempering of these nearby anatomical structures during STN-DBS may result in psychiatric symptoms called stimulation-induced transient nonmotor psychiatric symptoms (STPS), which are clinically manifested as depression [5], anxiety [6], apathy [7], explosive-aggressive behavior [8], manic episode [9], mirthful laughter [10], impulse control disorders [11], and so forth. Like other nonmotor functions, in some instances, STPS may have a drastic impact on a patient's life quality of life. [12]. It has been noted that the stimulation parameters which induce STPS are usually higher than normal. Decreasing stimulation intensity on the Implantable Pulsar Generator (IPG) may eliminate STPS. However, patients' motor functions may also be exacerbated. Changhai Hospital Neurosurgery Department conducts more than 160 DBS implantation surgeries every year. Since December 2014, the therapeutic center for Parkinson's disease in Changhai Hospital, Shanghai, China, has designed and implemented (1) an algorithm that identifies specific electrode contacts 


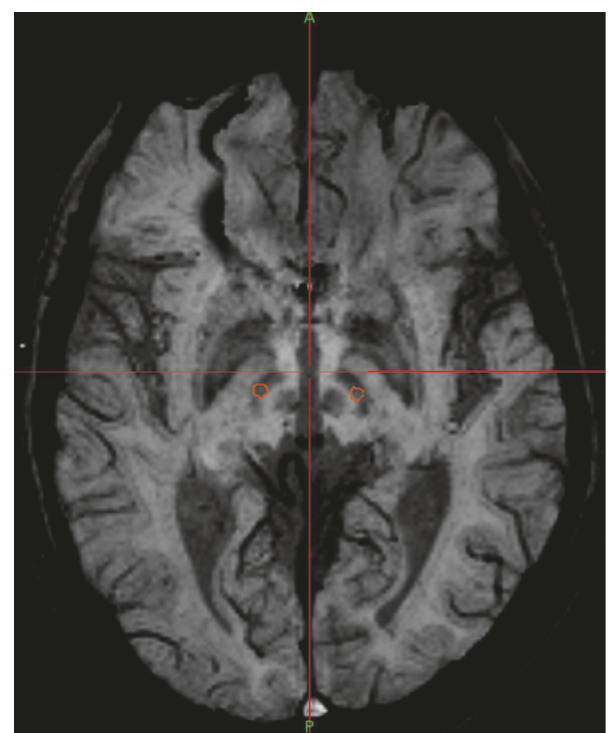

FIGURE 1: Merging SWI sequence with postoperation CT to determine the electrode position.

that induce STPS (2) a programming protocol to eliminate STPS. This study aim is to assess the effectiveness of these algorithms.

\section{Materials and Methods}

2.1. Patients. After acquiring approval from the Ethics Committee of Changhai Hospital, we retrospectively analyzed all patients with idiopathic Parkinson's disease who received clinical programming of implanted bilateral STN-DBS in the DBS programming clinic of Changhai Hospital, Shanghai, China, from January 1, 2015, to December 31, 2015. Patients who are being initiated on DBS, as well as those receiving follow-up programming sessions due to various reasons after achieving optimal stimulation parameters, were included in the present study. The UK PD Brain Bank diagnostic criteria were adopted for the diagnosis of idiopathic Parkinson's disease.

2.2. Mapping the Active Contact. If the active contact is not within the STN, the programming protocol may be less effective. Therefore we reviewed intraoperative MRI of all the patients with Leksell $G$ frame and indicator to confirm the electrode location. The Medtronic S7 Neuro Navigation System (Medtronic Navigation, Louisville, USA) was used to merge preoperative 3.0T-MRI images with postoperative CT images. The CT scan images were calibrated at $1 \mathrm{~mm}$ thickness and without pneumocranium (Figure 1). It was essential that the active contact center is placed within the boundaries of the STN. Patients with the active contact center outside STN boundary were excluded from this study.

2.3. Motor Assessment. Patient's motor function was graded using the Unified Parkinson's Disease Rating Scale Part III (UPDRS-III, 1998 edition). All patients included in this study were assessed preoperatively, postoperatively and then, finally, before and after the occurrence of STPS. All clinicians who administered the Unified Parkinson's Disease Rating Scale in this study are board-certified with the International Parkinson and Movement Disorder Society.

2.4. Cognitive and Psychiatric Assessment. All patients with STPS were enrolled in this study. Patients that had any mild psychiatric episodes that occurred more than twice as a result of programming (increasing) the electrode power parameters at home by oneself or caregivers were enrolled in this study. Episodes of acute psychiatric symptoms during or after the clinic programming procedures were also enlisted. Patients with psychiatric symptoms similar to the preoperative ones or had changed medication dose in less than one month were excluded. This was because it would be difficult to confirm whether the symptoms were induced by electric stimulation or not. So, only new postoperative psychiatric symptoms were regarded as suspicious STPS. The litmus test for STPS identification was defined by (1) psychiatric symptoms of patients which improved after decreasing stimulation intensity in 30 minutes; however, this could also confirm that the STPS was induced by stimulation because it occurred at the time when stimulation intensity was increased to improve the patient's motor functions, (2) patients who must have no similar history of psychiatric symptoms and upon clinical programming have shown marked improvement within three months without any change in the medication, and (3) identification of causative STPS active contact.

Cognitive and depressive symptoms were evaluated preoperatively and graded using Unified Parkinson's Disease Rating Scale Parts I and II (UPDRS-I and UPDRS-II), the Mini-Mental State Examination (MMSE), and the 17-item Hamilton Depression Scale (HAM-D-17). Mania type STPS was graded using the Young Mania Rating Scale (YMRS) [13]. The YMRS was calculated using patient collateral history from caregivers and direct observation by the physicians. Patients with STPS while being initiated on IPG were recorded using preoperative evaluation results only.

2.5. Assessment of STPS and Recording of Stimulation Parameters. Patient's collateral history regarding unusual psychiatric symptoms recorded in programming sessions was reviewed. Stimulus parameters (active, stimulating contacts, stimulating pattern, voltage/current, pulse width, and frequency) were recorded following the occurrence of STPS. Changes and the final status of stimulation parameters in programming sessions were also recorded. Electrode contacts inducing STPS and programming protocols were determined according to previously established flow diagrams (Figures 2 and 3). The disappearance of STPS was determined by no relapse of similar psychiatric symptoms for three months after programming.

2.6. Statistical Analysis. Paired-sample $t$-test was used to examine for changes of variables in UPDRS-III during clinical evaluation. $p<0.05$ was considered statistically significant. 


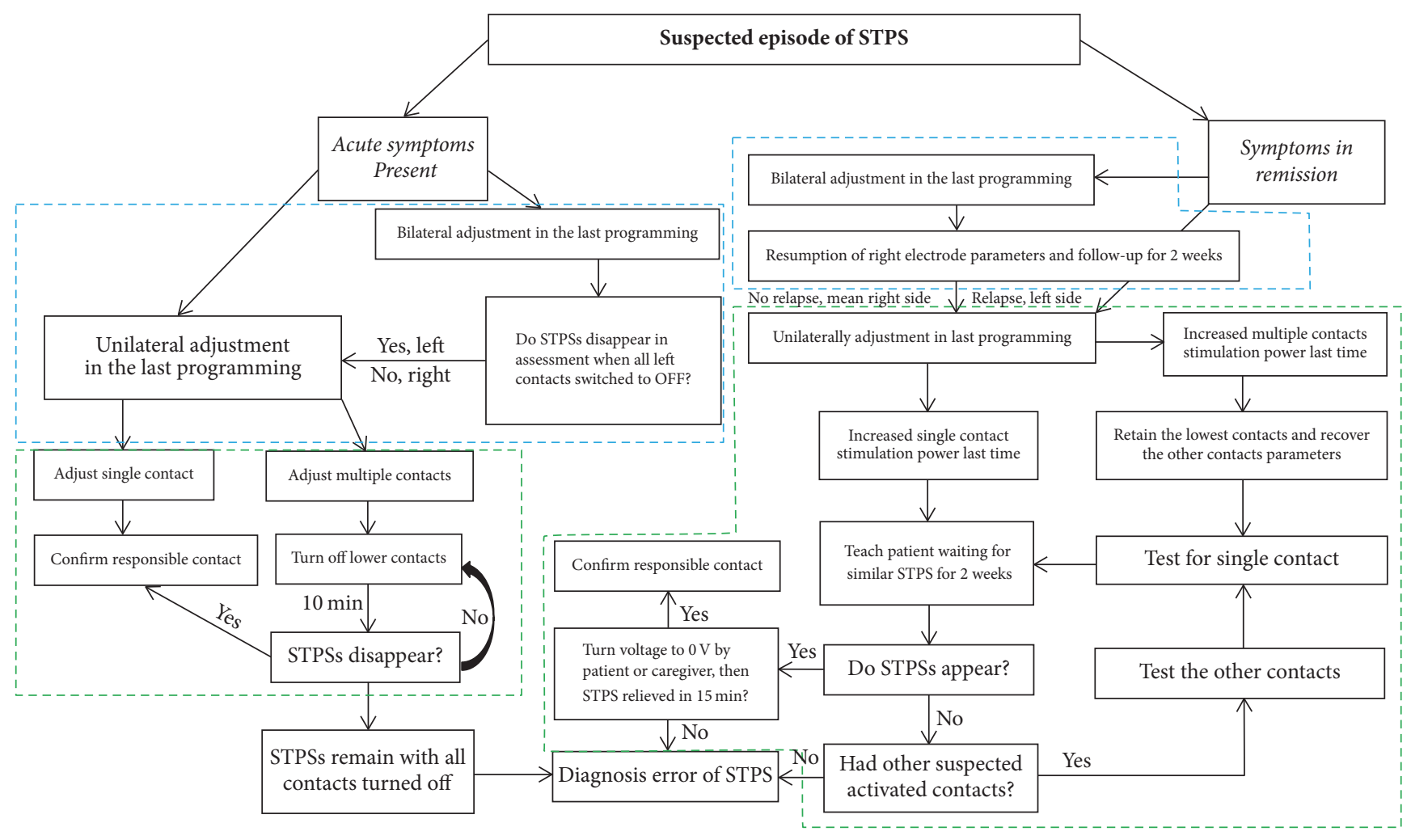

Description

- - Confirmation of electrode position

- - Confirmation of causative contact

FIGURE 2: Flow diagram to determine electrode contacts inducing STPS.

\section{Results}

3.1. Baseline Data. There were three groups of patients enrolled in this study: (1) the first group is comprised of 145 patients undergoing DBS initiation 1 month postoperatively for optimal programming parameter; (2) the second group had 231 patients who underwent DBS less than one year postoperatively; (3) finally, the third group had 166 patients who underwent DBS more than one year postoperatively. There were a total number of 1142 episodes of clinical programming for the purpose of adjusting the stimulation parameters in the second and third groups. There were 20 episodes of STPS that occurred in 11 patients ( 6 females and 5 males). The mean age at the time of DBS surgery was $63.45 \pm 8.27$ years. The mean duration between identification of Parkinsonian symptoms and DBS surgery was $11.91 \pm$ 3.33 years. Cognitive functional impairment was excluded in all patients. Only six patients received antidepressant drugs before and after surgery, while the rest of the patients had no obvious depression symptoms. Preoperatively, patients were given an average Levodopa Equivalent Daily Dose (LEDD) of $942.5 \pm 232.7 \mathrm{mg}$. Postoperatively, before the STPS occurred, the LEDD was reduced to $404.5 \pm 353.3 \mathrm{mg}$. By the time programming eliminated STPS at 3 months after programming, the LEDD had further reduced to $284.5 \pm 187.8 \mathrm{mg}$. The UPDRS-III improvement rate between postoperative (med-off time) status and preoperative (med-off time) status was $35.14 \sim 72.34 \%$ at 3 months after programming. When we compared the baseline data of STPS + patients versus STPS patients to characterize patients inherent risk factors for STPS, no significant statistical difference was found between groups (see Table 1).

3.2. STPS Occurrence. During the programming, 20 episodes of STPS occurred in 11 patients. These psychiatric symptoms consisted of four (04) episodes of crying in two (02) patients, four (04) episodes of inexplicable euphoria or mirthful laughter in two (02) patients, two (02) episodes of spatial disorientation in one patient, two (02) episodes of hallucination in one patient, and eight (08) episodes of hypomania in five (05) patients. In five (05) patients, STPS occurred during titration adjustment for optimal programming parameter in the first year after devices were implanted, while two (02) patients showed psychiatric symptoms two days after being started on IPG and discharged from the hospital. For patients who had implanted devices for more than one year, three (03) developed STPS during programming sessions. Manifestations of patients during STPS episodes 


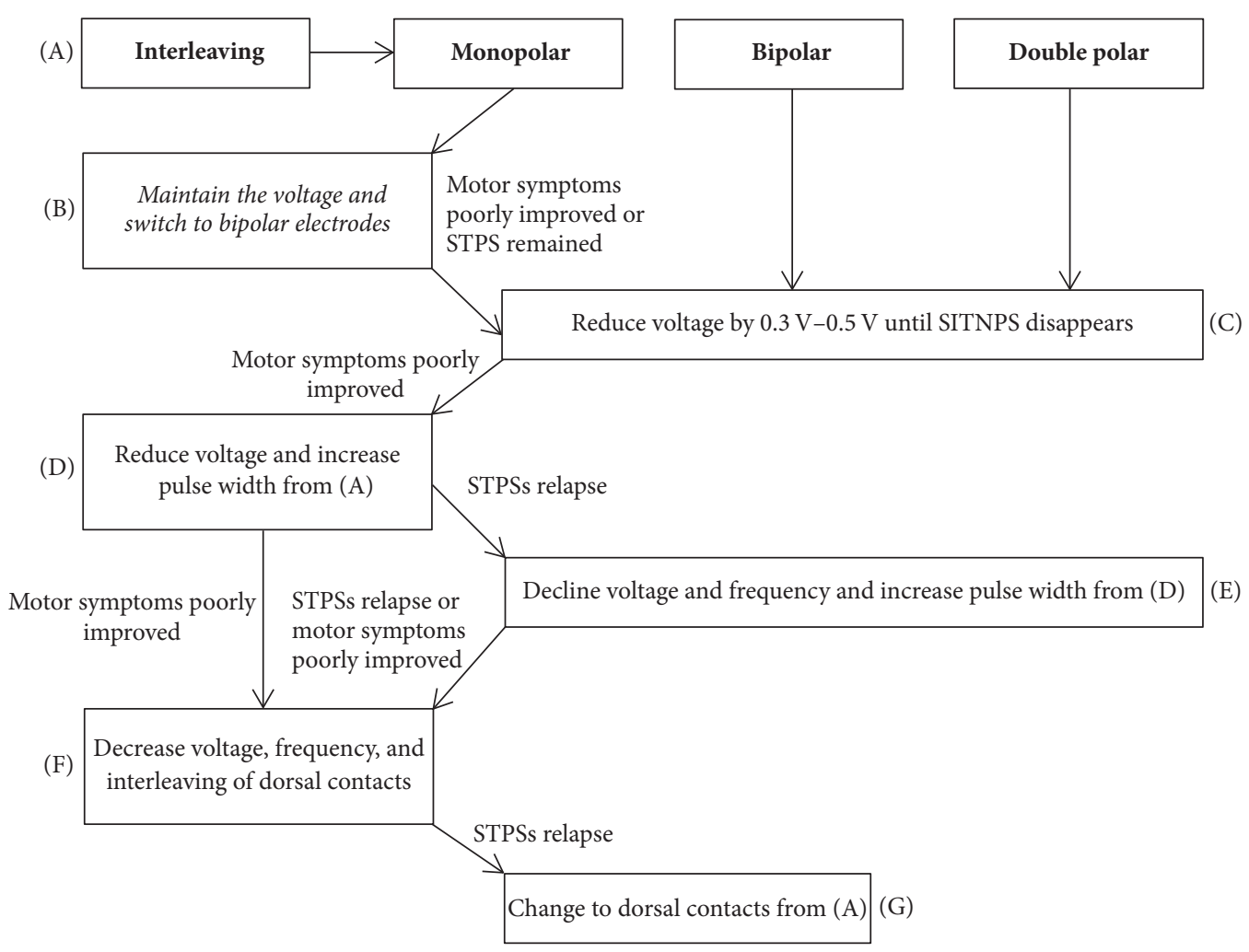

FIGURE 3: Programming protocol flow diagram (After determining STPS causative active contacts with Figure 2, (A) record the initial parameters and programming according to the stimulation mode; (D) recover the initial parameters and reduce voltage $0.3 \mathrm{~V}-0.5 \mathrm{~V}$ and increase pulse width by $10-20 \mu \mathrm{s}$; (E) if STPS relapsed, then reduce voltage $0.1-0.3 \mathrm{~V}$ and reduce frequency $10-30 \mathrm{~Hz}$, increase pulse width $10-20 \mu \mathrm{s}$, and keep the total electrical energy delivered (TEED) equal to the counterpart of (D), TEED $=\operatorname{voltage}^{2} \times$ frequency $\times$ pulse width/impedance; (F) decrease frequency to $125 \mathrm{~Hz}$ and decrease voltage $0.3-0.5 \mathrm{~V}$, and keep the TEED equal or slightly higher to the counterpart of (D) or (E); activate the other dorsal contact with interleaving mode.).

were described in Table 2. The stimulation parameters before the onset of STPS were listed in Table 3.

3.3. Programming of STPS and Motor Functions. Following the adjustment of stimulation parameters (Table 3), ten patients maintained the improvement of motor functions with psychiatric symptoms eliminated. The adjustments were based on the algorithms as follows: (1) the stimulation voltage decreased while the pulse width increased in 2 patients (numbers 1 and 11); (2) the voltage was maintained while switching to bipolar stimulation in 2 patients (numbers 2 and 7); (3) voltage and frequency were decreased with pulse width increased in 1 patient (number 9); (4) voltage and frequency were decreased while switching to interleaving stimulation in 3 patients (numbers 3,4 , and 10); (5) the activation contacts were replaced with dorsal ones in 2 patients (numbers 5 and 8). One patient (number 6), after multiple programming sessions, developed concurrent motor and nonmotor functions, and UPDRS-III score increased by 2 points under the final stimulation parameters by her choice (see details of the programming duration in Table 4). There were no statistical differences between the UPDRS-III under the final stimulation parameter (without STPSs) and optimum UPDRS-III under the STPSs $(26.45 \pm 10.59$ versus $26.45 \pm 10.17, p=1.000$ ).

\section{Discussion}

4.1. The Clinical Value of Programming Algorithms for STPS. Since the application of STN-DBS in the treatment of PD patients, there have been sporadic reports [10] noting STPSs, as one of the side effects. The development of these programming algorithms is aiming to reduce the ambiguity in the management of STPS. The ambiguity was on the predisposition of the following. (1) In earlier studies, STPS was implicated with stimulation of the medial and inferior part (limbic part) of STN. However, recent studies show their active contacts located in the dorsolateral (sensorimotor) area of STN [14] which also induces STPS. Because of this reason, the limbic and sensorimotor regions of the STN overlap were greater than what has been previously reported [15]. In this study, we also found that the active contacts which induced STPS were located at a medial and inferior part of the STN in seven (07) patients. In the other four (04) patients, the active contacts were located in the lateral part of STN which also induced STPS. This is similar to what was reported by Abulseoud and colleagues [14]. For this reason, we concluded that the side effects of STPS are difficult to avoid by just implanting contacts into dorsolateral STN. (2) Secondly, under the routine stimulation parameter, the spherical radius of active volume (without contacts volume) 


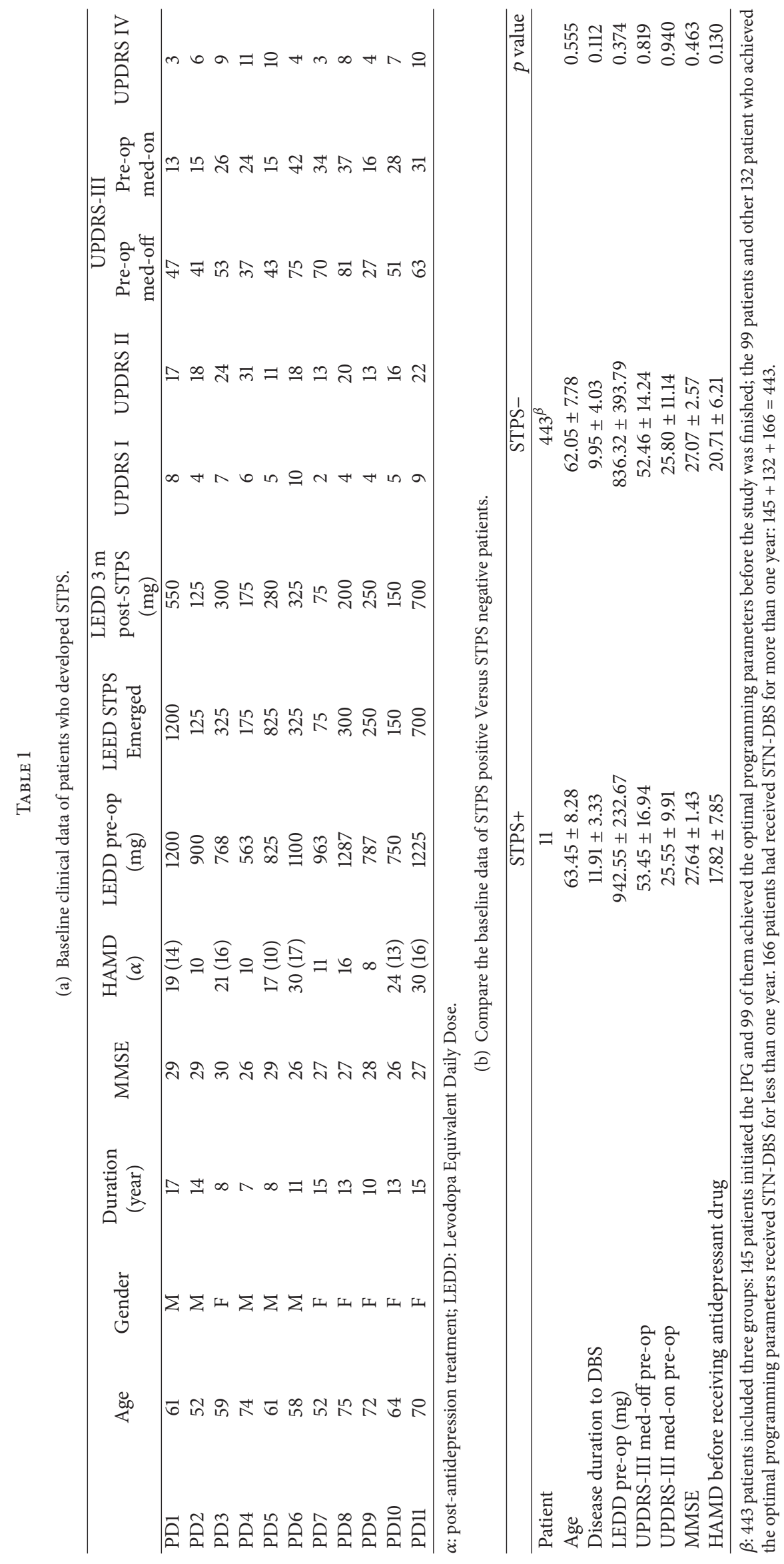




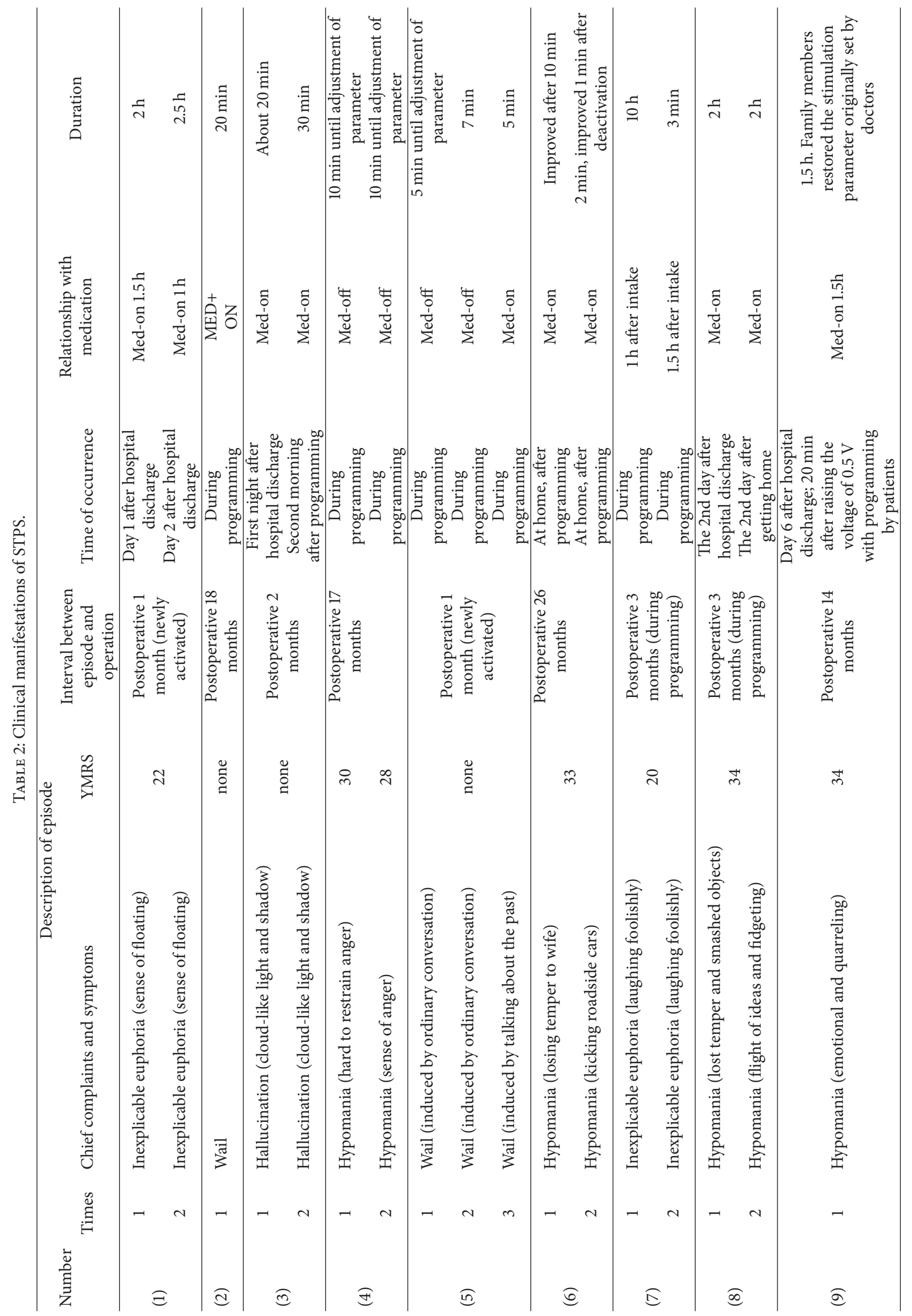




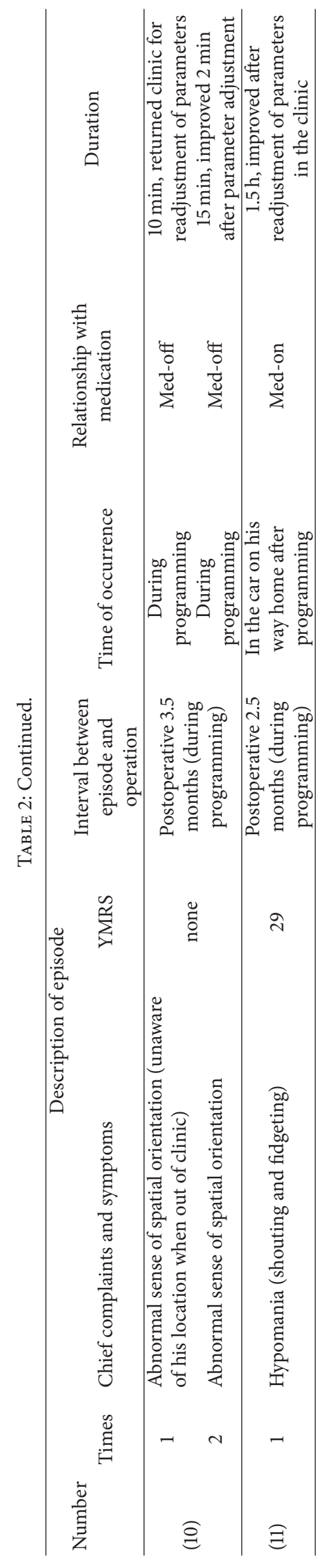




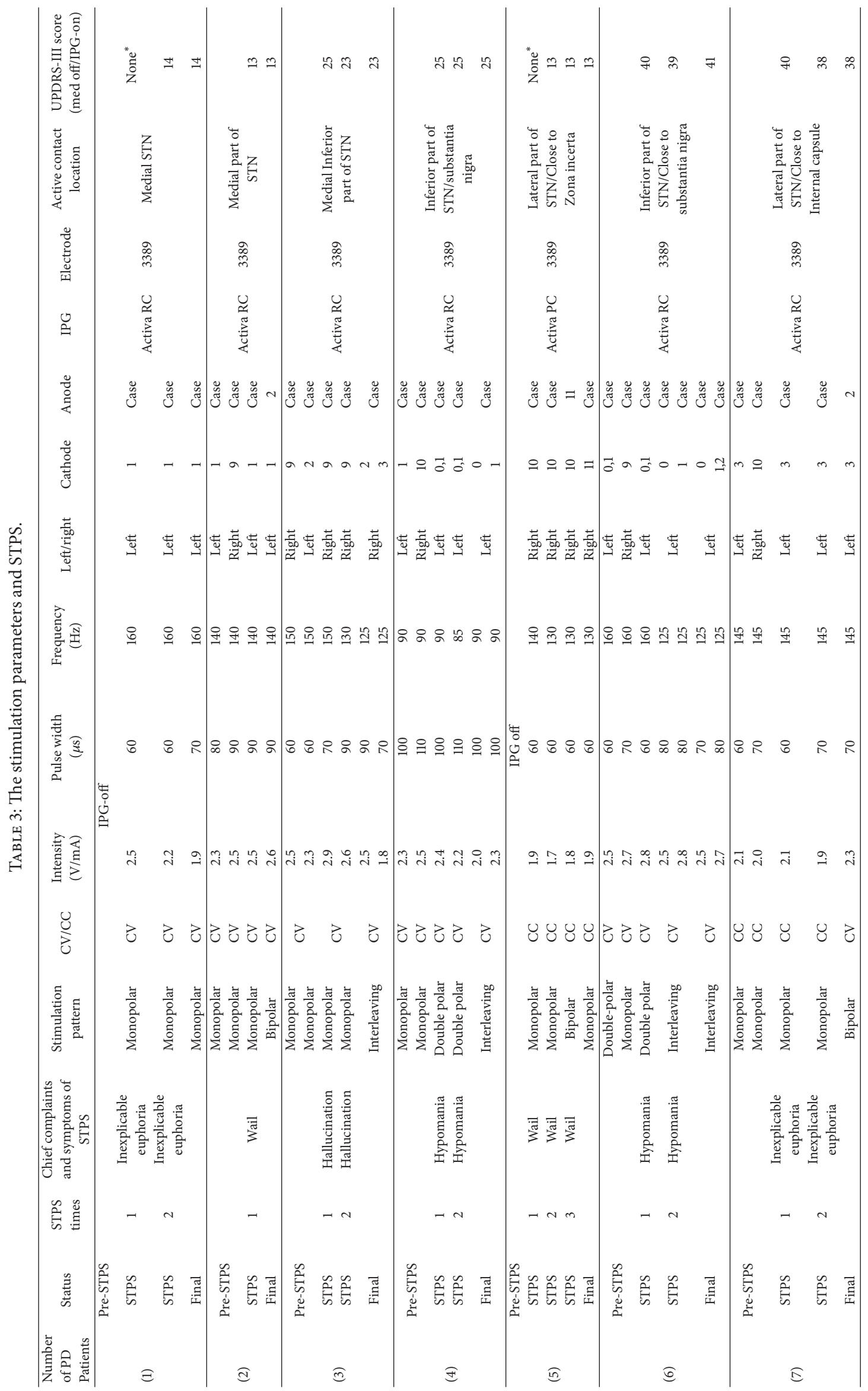




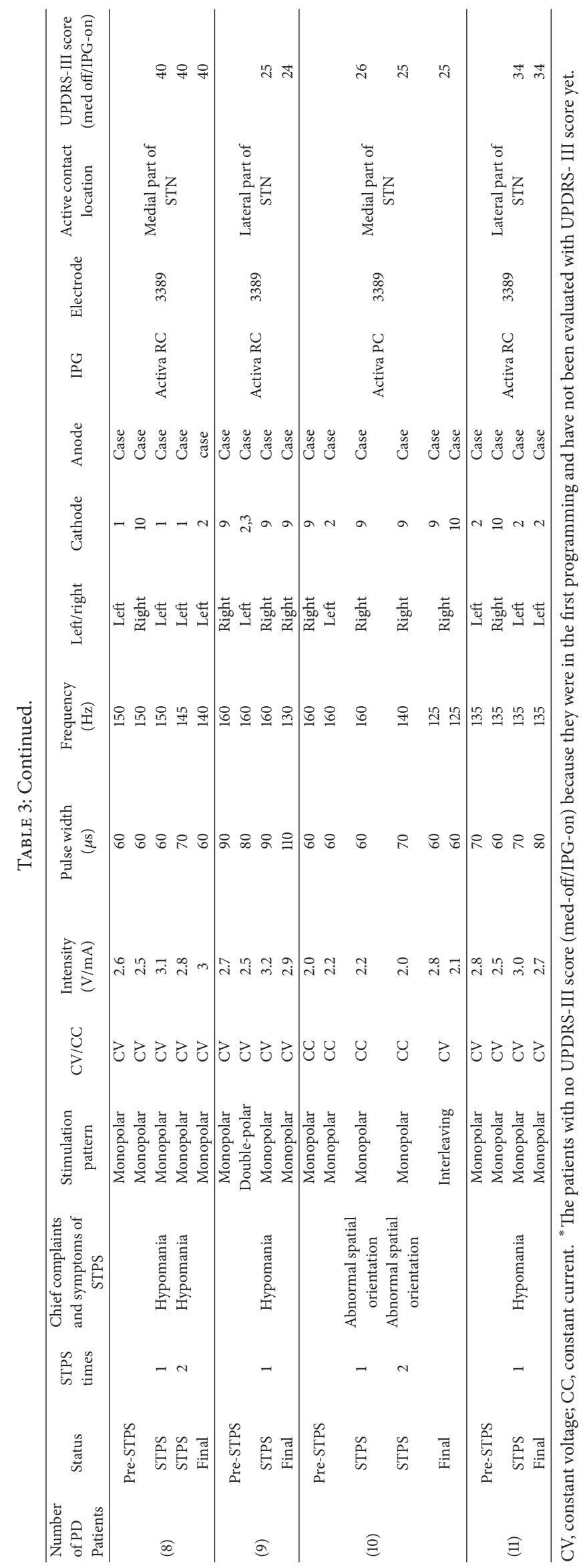




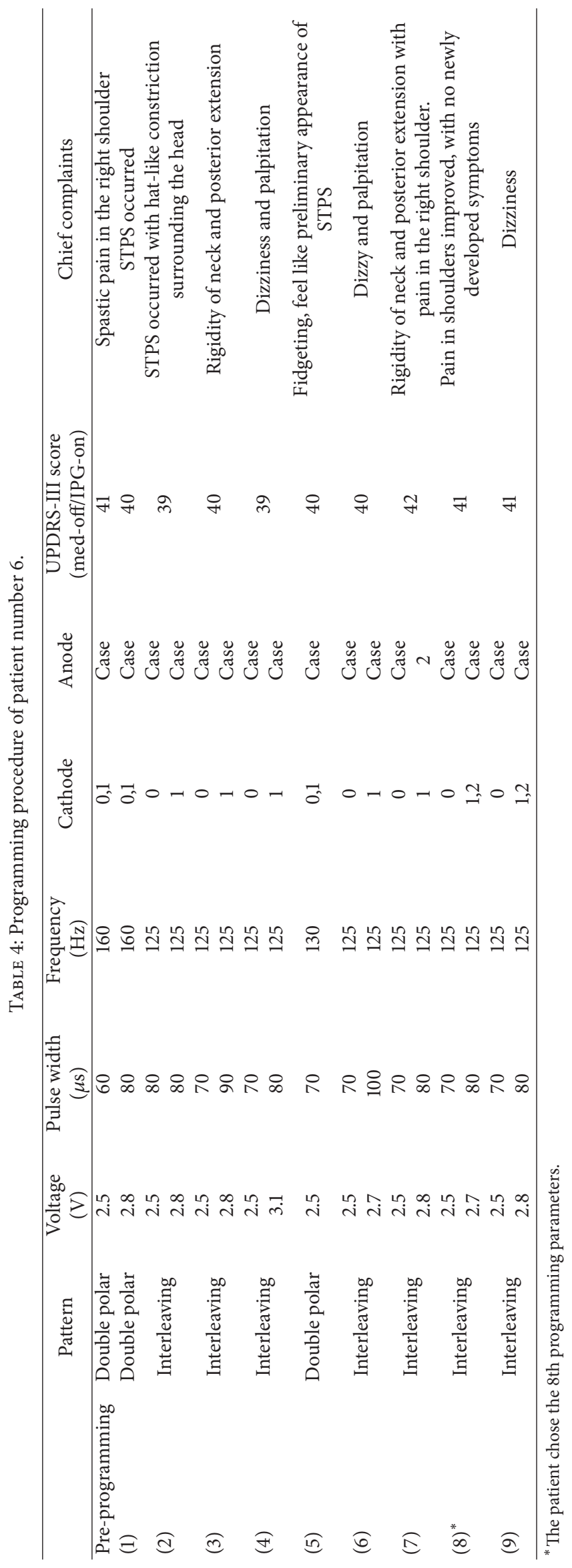


could reach to $3 \mathrm{~mm}$. As a result, the limbic part of STN could easily be affected and cause STPS. (3) Thirdly, even in the most experienced PD treatment center could not guarantee that both anticipated contacts location and curative stimulation effect could be satisfied. Meanwhile, STPS could be easily induced when increasing stimulation intensity or testing side effects.

China has the largest number of patients with PD in the world. In recent years, there have been more than 100 centers which carry out STN-DBS surgery for the treatment of PD. As a result, there is a strong likelihood of an increasing number of PD patients suffering STPS after STN-DBS programming. It is therefore very important to rapidly diagnose, locate contacts, and program parameters to eliminate STPS. It is also paramount to maintain the PD patient's optimal motor function according to algorithms set by programming doctors at different levels.

4.2. Confirmation of STPS and the Impact Factors. Psychiatric disorders are one of the symptoms manifested in PD patients. However, antiparkinsonism medications also manifest similar symptoms as drug side effects. Levodopa and dopaminergic drugs could induce dopamine dysregulation syndrome, hallucinations and psychosis, mania and hypomania, and impulse control disorders, which were similar to STPS [16]. The treatment of psychiatric symptoms induced by the above drugs is in the reduction of medication in most times. STPS is known to manifest similarly. Therefore the diagnosis of psychiatric symptoms induced by drug side effects or PD should not be misinterpreted for STPS. The diagnosis of STPS should only be made upon confirmation.

Patients in our study had neither any preexisting psychiatric symptoms nor their PD medication altered for than one month. Although preoperative LEDD given to our patients on average was more than the reported average dose before DBS surgery in previous studies $[17,18]$, the subsequent doses would reduce more than $50 \%$ similar to that reported by Jiang and colleagues [17]. Levodopa withdrawal symptoms mainly include apathy and depression. In our study, all 11 patients had no symptoms of apathy and depression. Moreover in the first three-month follow-up after STPS programming, except for the two patients who had STPS at IPG initiation, there was no change in the LEDD in the other patients (Table 1). We, therefore, deduced that the antiparkinsonism medication is not the main factor inducing psychiatric symptoms. Otherwise, some psychiatric symptoms may have reoccurred within the first 3 months of follow-up.

To avoid drugs side effect interference during DBS effect evaluation, all the patients were programming in the state of "off time." Patients were given daily doses of anti-Parkinson's disease drugs after the final stimulation parameters were set. To achieve the satisfactory therapeutic effects, the motor complications were evaluated during "on time" state. However, the interesting point is that 70\% (14/20) STPS occurred in "on time" state or over 1 hour after taking medications (though some patients had not felt medicine effect already); see Table 2. Whether medications for PD work with electrical stimulation to cause psychiatric symptoms or not needs further study. We excluded the effect of PD drugs by maintaining the same dosage that was given on the first occurrence of STPS. So we believe the electrical stimulation should be the main factor of psychiatric symptoms.

To improve the diagnosis of STPS, we developed the algorithm (Figure 2). The causative active contact of patients with acute symptoms of STPS could be easily identified using the flow chart. However, the causative active contact in patients with transient STPS symptoms was more difficult to identify. In the process of locating suspicious contacts in such patients, we do not recommend increasing stimulation intensity to induce STPS to identify contacts. The reason is that (1), theoretically, increasing stimulation intensity of any contact to a certain threshold, the volume of tissue activated (VTA) may affect limbic part of STN and induce STPS. So it may mislead the doctor to regard the contacts which were not the initial responsible contacts. The doctor may even locate wrong responsible electrodes and contacts. (2) If there are multiple active contacts (double polar, interleaving mode) on one side of the electrode, the adjacent contacts have a higher chance to induce STPS (although the ventral contacts are with a higher chance). If randomly increasing stimulation intensity of random contacts to induce STPS, it can also lead to wrong contact. (3) Intentionally induced STPS might cause patients extra distress, leading them to distrust doctors, increased complaints, and decreased satisfaction. Therefore, we recommend the utilization of the algorithm (Figure 2) to locate the contacts carefully and accurately. In our study, the active contacts which induced STPS were identified by using an algorithm (Figure 2) and eliminated by programming in all the 11 patients.

The clinical significance incidence rate of STPS which necessitated programming was about $2 \%$. This was significantly lower as compared to $28.6 \%$ in previous studies [14]. This difference may be attributed to the stimulation intensity used and the exclusion criteria we used to enroll patients with STPS symptoms in this study. In this study, the stimulating voltage was rarely more than $3.0 \mathrm{~V}$. However, the stimulating voltage did not exceed $3.3 \mathrm{~V}$.

We noted that time interval for the occurrence STPS fell within 48 hours after new settings of stimulation parameters. Therefore, the psychiatric and psychological status of patients should be thoroughly assessed in MED-ON/STIM-ON after each programming session before patients were discharged. It is also necessary to instruct the family to timely identify the occurrence of STPS, since some symptoms may not be recognized by patients themselves [19].

4.3. Principles of STPS Programming. We observed that STPS is likely to develop when programming clinicians increase the voltage or pulse width of the active contact. The increase in active contact voltage or pulse width gives patients a brief false sense of relief masked by improvement in motor functions. Even though the improvement of motor functions are most often minor ( 1 or 2 point differences in UPDRSIII), STPS set in as residual effects of the increase in voltage. Patients usually find themselves in a quagmire on whether to maintain improved motor functions or settle for removal of STPS and have less appealing motor functions. However, few reports were mentioning how to maintain the optimal 
improvement for motor functions while avoiding STPS at the same time.

We conducted a literature review using PubMed database of earlier authors who encountered and managed STPS while maintaining optimal motor functions. We designed a programming procedure and protocol to determine the active contacts that induce STPS to rapidly alleviate patients' psychiatric symptoms and maintain improved motor functions.

Previous reports [20] suggest that STPS was associated with high level of voltage. This suggests that the VTA might impact the limbic neuronal networks that induce STPS. They further reported that various programming methods decrease or change the VTA enabling eliminating STPS. These methods include (1) reducing voltage [20], (2) switching to bipolar stimulation [19], and (3) changing dorsal active contacts $[9,21,22]$.

Although there are many ways to improve STPS through programming, the order of choice must not be random but with precise principles and in chronological order (Figure 3). These principles include that (1) the solutions for eliminating STPS must be rapid; (2) simple and replicable; (3) and with minimal or no STPS reoccurrence and (4) must preserve optimal motor functions.

There are six methods to adjust the stimulation parameter: (1) bipolar stimulation, (2) reducing voltage by $0.3 \mathrm{~V}-$ $0.5 \mathrm{~V}$ until SITNPS disappears; (3) decreasing of voltage and increasing the pulse width; (4) declining voltage and frequency and increasing pulse width; (5) interleaving of original and dorsal contacts; (6) changing the active contacts to dorsal the position.

4.3.1. Bipolar Stimulation. The first option is bipolar stimulation. We choose to select bipolar stimulation to easily set stimulation parameters and quickly narrow the sphere VTA (Figure 3). This could promote STPS regression and reduce recurrence. However, due to the rapid narrowing of VTA, it often leads to the aggravation of motor functions.

4.3.2. Reducing Voltage. We reduced the voltage on the basis of initial stimulation parameter (with STPS). This adjustment can also quickly reduce the VTA and intensity of the activation domain.

\subsubsection{Decreasing of Voltage and Increasing the Pulse Width.} The adjustment of programming parameters mentioned above may reduce activation domain [23] while in the meantime declining the stimulating intensity of motor functionsrelated neural pathways [24]. Therefore a slight increase in the pulse width to maintain stimulation intensity in the narrowed VTA might be needed when the voltage is decreased. Some inexperienced programming physicians may conceive that it is hard to modulate pulse width appropriately. It will be easier to operate according to the formula of total electrical energy delivered (TEED) to calculate how to increase the pulse width according to the voltage reduced (new TEED should be not more than the original one with STPS).

4.3.4. Declining Voltage and Frequency and Increasing Pulse Width. If necessary, we can even adjust the frequency
$(+5-10 \mathrm{~Hz})$ to fit the change of the voltage and pulse width, to achieve the appropriate stimulation intensity to eliminate STPS while maintaining the improvement of motor functions. In addition to reducing the stimulation frequency to reduce intensity, interleaving technology could be used when adjusting the voltage.

4.3.5. Interleaving of Original and Dorsal Contacts. The adjustment of the pulse width is critical in improving patient's optimal movement symptom after the frequency has reduced to $125 \mathrm{~Hz}$. In the case where the active contact responsible for inducing STPS was ventrally positioned, stimulation parameters remain unaltered. Subsequently, the dorsally located contacts are activated into interleaving stimulation mode. This adjustment has proven to maintain optimal motor function.

4.3.6. Changing the Active Contacts to Dorsal the Position. In isolated patients, motor functions reacted better to stimulation frequency than the later adjustment. As reported, the contacts located anterior, medial, and inferior to STN were easier to precipitate STPS [14]; thus if using the above-mentioned scheme cannot eliminate STPS and achieve optimal improvement of motor functions at the same time, we change the active contact to more dorsal position to acquire a larger range in the adjustment of stimulation parameters. If the inactive contacts were suspended for a long time, curative and side effects might change from previous stimulation record and need retitration which is time-consuming. Also, after the inactivated contacts have been activated, the contact impedance may change with time causing instability of symptom control among patients [25]. We recommend that the interleaving mode generates two isolated VTA: the ventral and dorsal contact. The ventral contact maintains the previous stimulation parameters before the occurrence of STPS. We activate the dorsal contact by gradually increasing the voltage and pulse width to archive optimal motor function [26]. In contrast to the COMPARE trial [27], we have put in consideration preserving patient's motor function while eliminating STPS. Therefore the use of interleaving stimulation was our priority than changing the active contact to dorsal one.

4.4. Patient Follow-Up. The impedance of the active contacts reduces over time [25]. Therefore, after STPS programming, patients needed to be followed up for a period to ensure no appearance of similar psychiatric symptoms. STPS was considered eliminated after clinical programming at three (03) months. In the event there was a need to adjust the intensity of stimulation upwards, increasing the width of pulse was given priority. This is because the expansion of the activated domain was not as significant as that of the voltage [28].

4.5. Limitations. The limitations of the study are inadequate sample size and limited categories of STPS. Additionally, the flow diagrams adopted in this study are limited to Medtronic Activa PC and RC models. This was a single center study. 


\section{Conclusion}

The stimulation contacts in STPS could be determined with use of flow diagram. Appropriate programming could remove STPS while maintaining optimal improvement of motor functions for most patients.

\section{Additional Points}

Recommendations. The flow diagrams for patients with other models of IPG should be adjusted accordingly. A metaanalysis could increase the sample size and attain statistical significance.

\section{Disclosure}

This work was presented as a poster at the 2017 Chinese Medical Association of Nerve Regulation Professional Committee Annual Meeting and the 8th Session of the Chinese Nerve Regulation Conference. Xi Wu and Yiqing Qiu are co-first authors.

\section{Conflicts of Interest}

The authors declare that they have no conflicts of interest.

\section{Acknowledgments}

This work was supported by the National Key Research and Development Program of China (2016YFC0105900).

\section{References}

[1] A. Castrioto, A. M. Lozano, Y.-Y. Poon, A. E. Lang, M. Fallis, and E. Moro, "Ten-year outcome of subthalamic stimulation in Parkinson disease: A blinded evaluation," Archives of Neurology, vol. 68, no. 12, pp. 1550-1556, 2011.

[2] M. G. Rizzone, A. Fasano, A. Daniele et al., "Long-term outcome of subthalamic nucleus DBS in Parkinson's disease: from the advanced phase towards the late stage of the disease?" Parkinsonism and Related Disorders, vol. 20, no. 4, pp. 376-381, 2014.

[3] M. L. Janssen, A. A. Duits, A. M. Tourai et al., "Subthalamic Nucleus High-Frequency Stimulation for Advanced Parkinson's Disease: Motor and Neuropsychological Outcome after 10 Years," Stereotactic and Functional Neurosurgery, vol. 92, no. 6, pp. 381-387, 2014.

[4] P. J. Rossi, A. Gunduz, and M. S. Okun, "The Subthalamic Nucleus, Limbic Function, and Impulse Control," Neuropsychology Review, vol. 25, no. 4, pp. 398-410, 2015.

[5] A. Funkiewiez, C. Ardouin, E. Caputo et al., "Long term effects of bilateral subthalamic nucleus stimulation on cognitive function, mood, and behaviour in Parkinson's disease," Journal of Neurology, Neurosurgery \& Psychiatry, vol. 75, no. 6, pp. 834839, 2004.

[6] H. J. Kim, B. S. Jeon, and S. H. Paek, "Nonmotor functions and Subthalamic Deep Brain Stimulation in Parkinson's Disease," Journal of Movement Disorders, vol. 8, no. 2, pp. 38-91, 2015.
[7] D. Drapier, S. Drapier, P. Sauleau et al., "Does subthalamic nucleus stimulation induce apathy in Parkinson's disease?" Journal of Neurology, vol. 253, no. 8, pp. 1083-1091, 2006.

[8] M. Sensi, R. Eleopra, M. A. Cavallo et al., "Explosive-aggressive behavior related to bilateral subthalamic stimulation," Parkinsonism and Related Disorders, vol. 10, no. 4, pp. 247-251, 2004.

[9] D. Raucher-Chéné, C.-L. Charrel, A. D. de Maindreville, and F. Limosin, "Manic episode with psychotic symptoms in a patient with Parkinson's disease treated by subthalamic nucleus stimulation: Improvement on switching the target," Journal of the Neurological Sciences, vol. 273, no. 1-2, pp. 116-117, 2008.

[10] P. Krack, R. Kumar, C. Ardouin et al., "Mirthful laughter induced by subthalamic nucleus stimulation," Movement Disorders, vol. 16, no. 5, pp. 867-875, 2001.

[11] J. Volkmann, C. Daniels, and K. Witt, "Neuropsychiatric effects of subthalamic neurostimulation in Parkinson disease," Nature Reviews Neurology, vol. 6, no. 9, pp. 487-498, 2010.

[12] D. Floden, S. E. Cooper, S. D. Griffith, and A. G. Machado, "Predicting quality of life outcomes after subthalamic nucleus deep brain stimulation," Neurology, vol. 83, no. 18, pp. 1627-1633, 2014.

[13] R. C. Young, J. T. Biggs, V. E. Ziegler, and D. A. Meyer, "A rating scale for mania: reliability, validity and sensitivity," The British Journal of Psychiatry, vol. 133, pp. 429-435, 1978.

[14] O. A. Abulseoud, A. Kasasbeh, H.-K. Min et al., "Stimulationinduced transient nonmotor psychiatric symptoms following subthalamic deep brain stimulation in patients with Parkinson's disease: Association with clinical outcomes and neuroanatomical correlates," Stereotactic and Functional Neurosurgery, vol. 94, no. 2, pp. 93-101, 2016.

[15] P. Justin Rossi, C. Peden, O. Castellanos et al., "The human subthalamic nucleus and globus pallidus internus differentially encode reward during action control," Human Brain Mapping, vol. 38, no. 4, pp. 1952-1964, 2017.

[16] I. Beaulieu-Boire and A. E. Lang, "Behavioral effects of levodopa," Movement Disorders, vol. 30, no. 1, pp. 90-102, 2015.

[17] L.-L. Jiang, J.-L. Liu, X.-L. Fu et al., "Long-term efficacy of subthalamic nucleus deep brain stimulation in parkinson's disease: A 5-year follow-up study in China," Chinese Medical Journal, vol. 128, no. 18, pp. 2433-2438, 2015.

[18] J. Li, Y. Zhang, and Y. Li, "Long-term follow-up of bilateral subthalamic nucleus stimulation in Chinese Parkinson's disease patients," British Journal of Neurosurgery, vol. 29, no. 3, pp. 329333, 2015

[19] T. S. Mandat, T. Hurwitz, and C. R. Honey, "Hypomania as an adverse effect of subthalamic nucleus stimulation: Report of two cases," Acta Neurochirurgica, vol. 148, no. 8, pp. 895-898, 2006.

[20] A. Chopra, S. J. Tye, K. H. Lee et al., "Voltage-dependent mania after subthalamic nucleus deep brain stimulation in Parkinson's disease: A case report," Biological Psychiatry, vol. 70, no. 2, pp. e5-e7, 2011.

[21] T. T. Ugurlu, G. Acar, F. Karadag, and F. Acar, "Manic episode following deep brain stimulation of the subthalamic nucleus for Parkinson's disease: A case report," Turkish Neurosurgery, vol. 24, no. 1, pp. 94-97, 2014.

[22] L. Mallet, M. Schüpbach, K. N’Diaye et al., "Stimulation of subterritories of the subthalamic nucleus reveals its role in the integration of the emotional and motor aspects of behavior," Proceedings of the National Academy of Sciences of the United States of America, vol. 104, no. 25, pp. 10661-10666, 2007. 
[23] B. Mädler and V. A. Coenen, "Explaining clinical effects of deep brain stimulation through simplified target-specific modeling of the volume of activated tissue," American Journal of Neuroradiology, vol. 33, no. 6, pp. 1072-1080, 2012.

[24] N. Yousif, R. Bayford, P. G. Bain, and X. Liu, "The peri-electrode space is a significant element of the electrode-brain interface in deep brain stimulation: A computational study," Brain Research Bulletin, vol. 74, no. 5, pp. 361-368, 2007.

[25] C. J. Hartmann, L. Wojtecki, J. Vesper et al., "Long-term evaluation of impedance levels and clinical development in subthalamic deep brain stimulation for Parkinson's disease," Parkinsonism and Related Disorders, vol. 21, no. 10, article no. 2753, pp. 1247-1250, 2015.

[26] S. Miocinovic, P. Khemani, R. Whiddon et al., "Outcomes, management, and potential mechanisms of interleaving deep brain stimulation settings," Parkinsonism and Related Disorders, vol. 20, no. 12, pp. 1434-1437, 2014.

[27] M. S. Okun, H. H. Fernandez, W. u. SS et al., "Cognition and mood in Parkinson's disease in subthalamic nucleus versus globus pallidus interna deep brain stimulation: the COMPARE trial," Annals of Neurology, vol. 65, no. 5, pp. 586-595, 2009.

[28] A. Chaturvedi, J. L. Luján, and C. C. McIntyre, "Artificial neural network based characterization of the volume of tissue activated during deep brain stimulation," Journal of Neural Engineering, vol. 10, no. 5, Article ID 056023, 2013. 


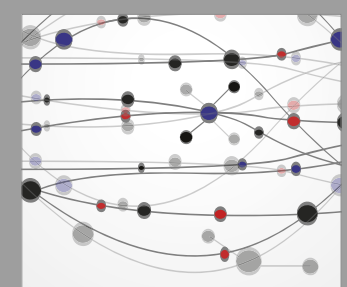

The Scientific World Journal
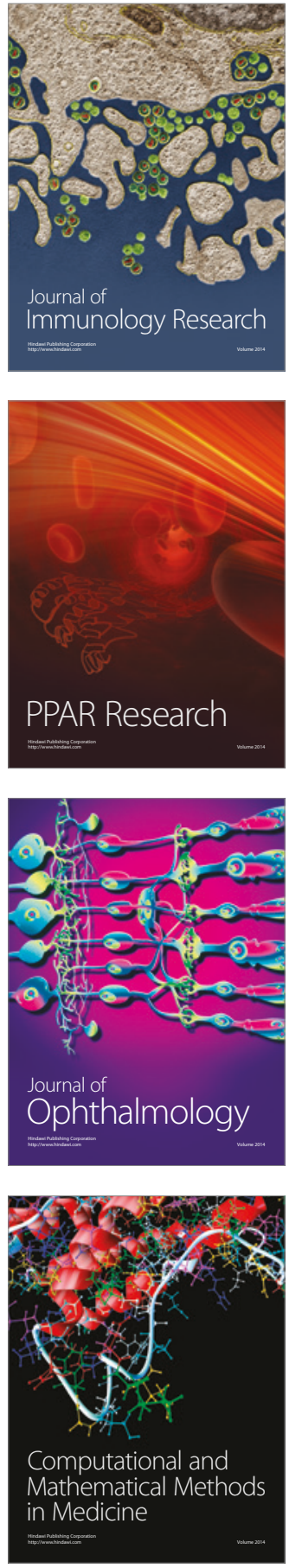

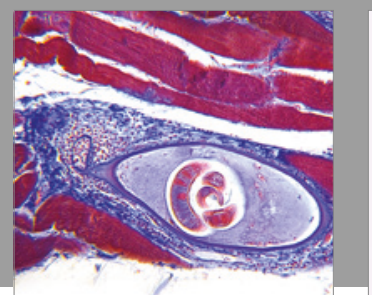

Gastroenterology Research and Practice
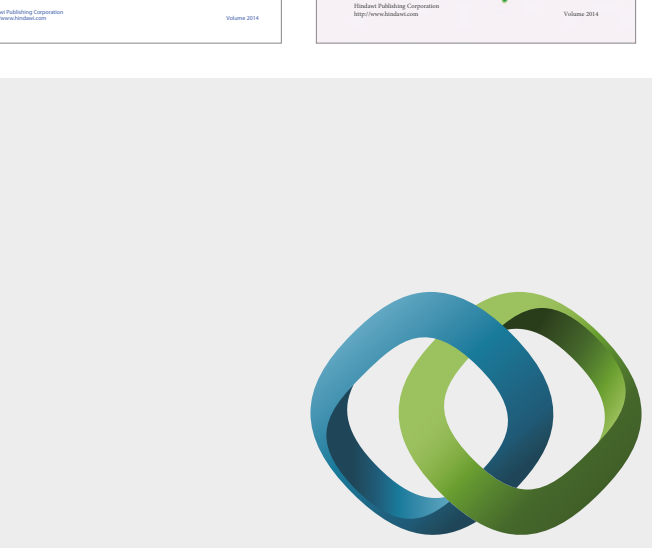

\section{Hindawi}

Submit your manuscripts at

https://www.hindawi.com
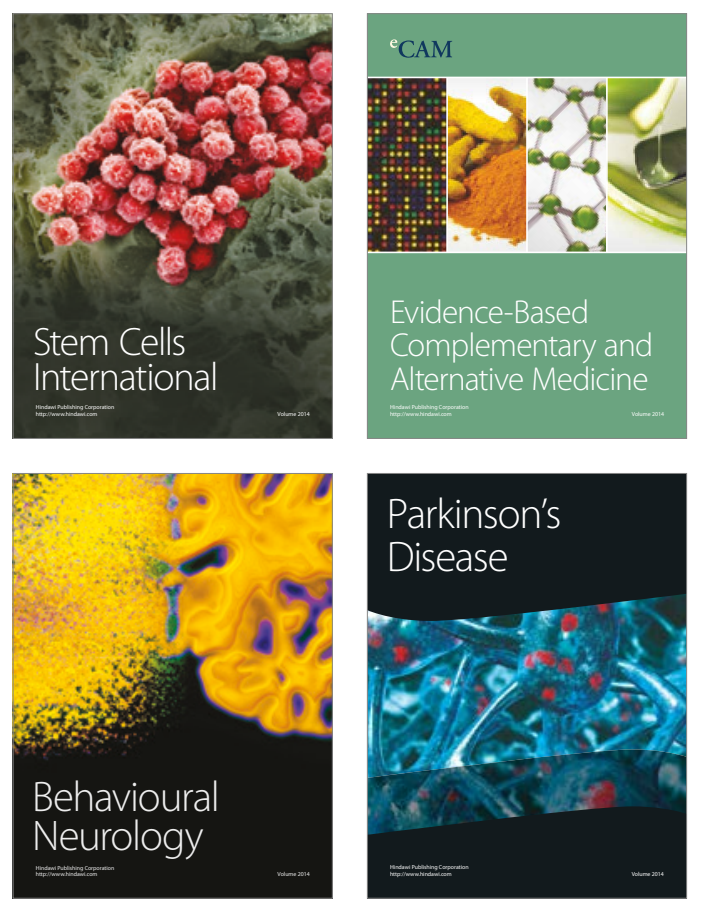
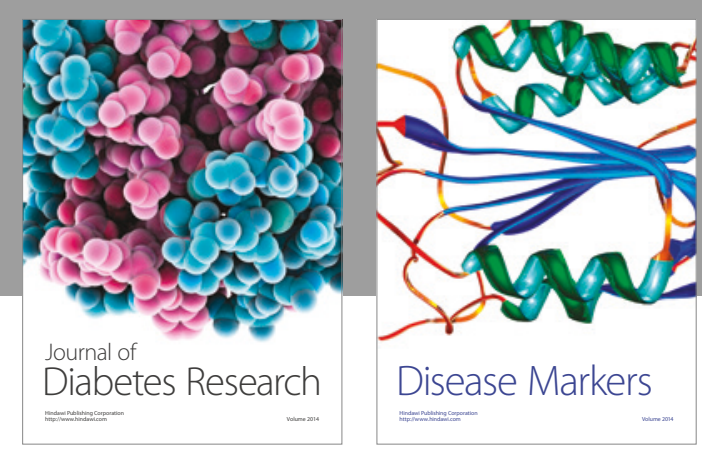

Disease Markers
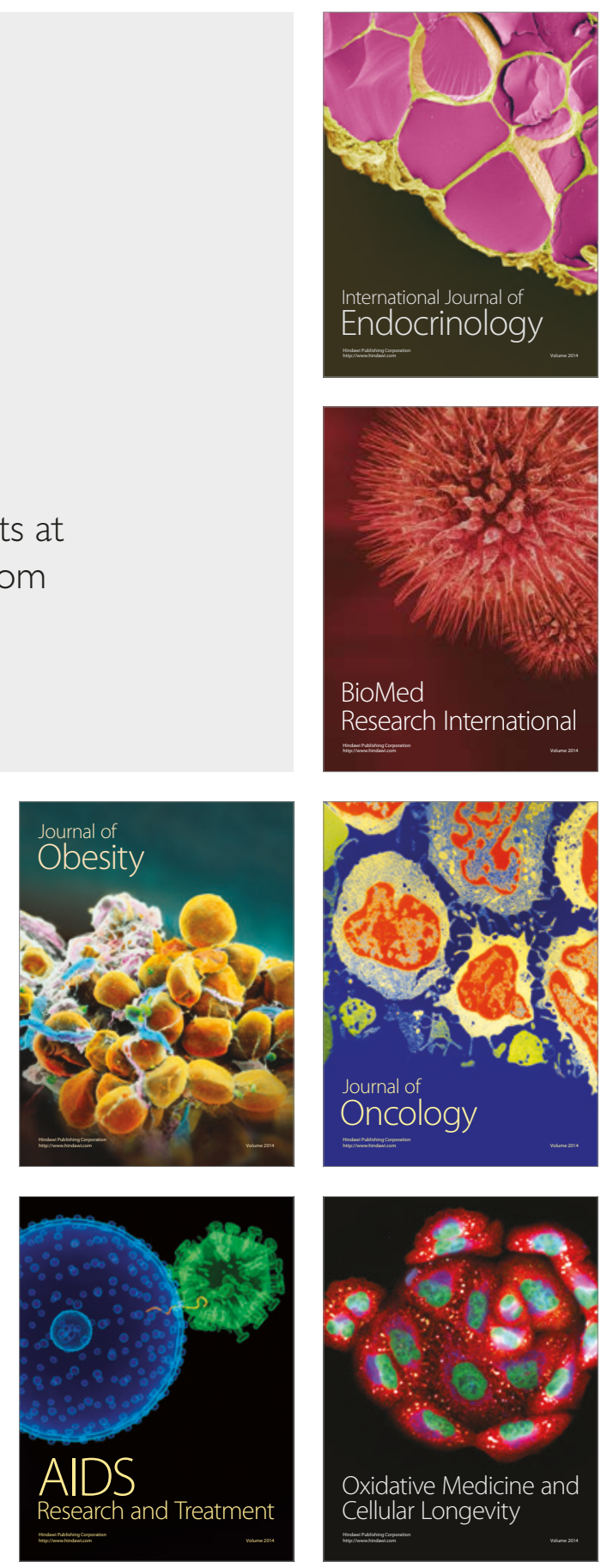